\title{
Exact Solution of Klein-Gordon with the Pöschl-Teller Double-Ring-Shaped Coulomb Potential
}

\author{
H. HASSANABADI ${ }^{a, *}$, A.N. IKOT ${ }^{b}$ AND S. ZARRINKAMAR ${ }^{c}$ \\ ${ }^{a}$ Department of Basic Sciences, Shahrood University, Shahrood, Iran \\ ${ }^{b}$ Department of Physics, University of Port Harcourt, Choba, PMB 5323, Port Harcourt, Nigeria \\ ${ }^{c}$ Department of Basic Sciences, Garmsar Branch, Islamic Azad University, Garmsar, Iran
}

(Received October 13, 2013; in final form May 11, 2014)

\begin{abstract}
Analytical solution of the Klein-Gordon equation under the equal scalar and vector Pöschl-Teller double-ring-shaped Coulomb potentials is obtained. We have used the Nikiforov-Uvarov method in our calculations. The radial wave function in terms of the Laguerre polynomials is presented and the angular wave functions are expressed in terms of the Jacobi polynomials. We have also considered some special cases of the Pöschl-Teller double-ring-shaped Coulomb potential and represented the energy eigenvalues and the corresponding wave functions.
\end{abstract}

DOI: 10.12693 /APhysPolA.126.647

PACS: 03.65.Fd, 03.65.Pm, 03.65.Ca, 03.65.Ge

\section{Introduction}

Although the central potentials have had great predictions in various physical fields, they fail in some places including in the study of deformed nuclei or particular molecular configurations like benzenes. This has motivated a modification of successful central interactions proper angle-dependent ones. On the other hand, the Pöschl-Teller [1] stands as a good candidate in nuclear and molecular physics [2-17]. It has also well accounted for many recent studies in the quantum potential model such as excitons, quantum wires, and dots [18-22]. Such encouraging results have arisen some studies on the potential within the frame work of common wave equations of both nonrelativistic and relativistic wave equations, i.e. including the Schrödinger, Dirac, Duffin-KemmerPetiau (DKP), or Klein-Gordon equations [23-38]. In such studies, except for the $s$-wave solutions, approximate techniques are inevitable due to the inverse square centrifugal term. In our study, we consider the KleinGordon equation [39] under the Pöschl-Teller double-ring-shaped Coulomb potential [40,41]:

$$
V(r, \theta, \varphi)=V(r)+\frac{V(\theta)}{r^{2}}+\frac{V(\varphi)}{r^{2} \sin ^{2} \theta},
$$

where

$$
\begin{aligned}
& V(r)=-\frac{\beta}{r}, \quad V(\theta)=\frac{b}{\sin ^{2} \theta}+\frac{A(A-1)}{\cos ^{2} \theta}, \\
& V(\varphi)=\frac{\alpha^{2} D(D-1)}{\sin ^{2}(\alpha \varphi)}+\frac{\alpha^{2} C(C-1)}{\cos ^{2}(\alpha \varphi)},
\end{aligned}
$$

and $A, C, D>1, \beta>0, b \geq 0, \alpha=1,2,3 \ldots$ are real positive parameters. The Coulomb, Hartmann, double-ring-shaped Coulomb potentials are special cases of the Pöschl-Teller double-ring-shaped Coulomb potential. For $A=C=D=1$ and $b=0$ this potential reduces to the Coulomb potential. When $A=C=D=1$, the

* corresponding author; e-mail: h.hasanabadi@shahroodut.ac.ir potential reduces to the Hartmann potential which was introduced in quantum chemistry to describe ring-shaped molecules like benzene. Bound state of the Dirac equation for the Hartmann potential has been investigated in [41]. For $C=D=1$, it changes into the double-ring-shaped Coulomb potential. In Refs. [41, 42], the bound states of Schrödinger and Klein-Gordon equations for double-ring-shaped oscillator potential have been obtained. We organize our work as follows. In Sect. 2, we review the angular and radial parts of the Klein-Gordon equation with Pöschl-Teller double-ring-shaped Coulomb potential. In Sects. 2.1, 2.2, and 2.3, the azimuthal, polar and radial parts of the equation are respectively solved via the Nikiforov-Uvarov (NU) method and exact eigenfunctions and eigenenergies are presented. We give the solutions of some special cases of the Pöschl-Teller double-ring-shaped Coulomb potential in Sect. 3, and finally conclusion appears in Sect. 4 .

\section{Solution of the Klein-Gordon equation with Pöschl-Teller double-ring-shaped Coulomb potential}

The Klein-Gordon equation in the presence of vector potential $V(r)$ and scalar potential $S(r)$ is written as $(\hbar=c=1)[11]:$

$$
\left[\hat{p}^{2}+(M+S(r))^{2}-(E-V(r))^{2}\right] \psi(\boldsymbol{r})=0,
$$

where $\hat{p}$ is the momentum operator, $E$ is the energy, and $M$ is the rest mass of the particle. Under the condition of equal scalar and vector potentials, Eq. (3) becomes

$$
\begin{aligned}
& {\left[\hat{p}^{2}+\left(M^{2}-E^{2}\right)+2(M+E)\left(-\frac{\beta}{r}+\frac{1}{r^{2}}\left(\frac{b}{\sin ^{2} \theta}\right.\right.\right.} \\
& \left.+\frac{A(A-1)}{\cos ^{2} \theta}\right)+\frac{1}{r^{2} \sin ^{2} \theta}\left(\frac{\alpha^{2} D(D-1)}{\sin ^{2}(\alpha \varphi)}\right. \\
& \left.\left.\left.+\frac{\alpha^{2} C(C-1)}{\cos ^{2}(\alpha \varphi)}\right)\right)\right] \psi(\boldsymbol{r})=0 .
\end{aligned}
$$

By considering the wave function as below [40]: 
$\psi(\boldsymbol{r})=\frac{R(r)}{r} \frac{H(\theta)}{\sin ^{\frac{1}{2}} \theta} K(\varphi)$,

and substituting Eq. (5) into Eq. (4), after separation of variables, we obtain the decoupled differential equations for $R(r), H(\theta)$ and $K(\varphi)$ as

$$
\begin{aligned}
& -\frac{\mathrm{d}^{2} R_{n, l, m}(r)}{\mathrm{d} r^{2}}+\left[\left(l^{2}-\frac{1}{4}\right) \frac{1}{r^{2}}-2 \beta\left(M+E_{n, l, m}\right) \frac{1}{r}\right] R_{n, l, m}(r)=\left(E_{n, l, m}^{2}-M^{2}\right) R_{n, l, m}(r), \\
& -\frac{\mathrm{d}^{2} H_{l}(\theta)}{\mathrm{d} \theta^{2}}+\left[\frac{m^{2}+2 b\left(M+E_{n, l, m}\right)-\frac{1}{4}}{\sin ^{2} \theta}+\frac{2 A(A-1)\left(M+E_{n, l, m}\right)}{\cos ^{2} \theta}\right] H_{l}(\theta)=l^{2} H_{l}(\theta), \\
& -\frac{\mathrm{d}^{2} K_{m}(\varphi)}{\mathrm{d} \varphi^{2}}+2\left(M+E_{n, l, m}\right)\left[\frac{\alpha^{2} D(D-1)}{\sin ^{2}(\alpha \varphi)}+\frac{\alpha^{2} C(C-1)}{\cos ^{2}(\alpha \varphi)}\right] K_{m}(\varphi)=m^{2} K_{m}(\varphi),
\end{aligned}
$$

where $m$ and $l$ are the separation constants.

\subsection{Solution of the azimuthal part}

To obtain the solution of Eq. (8), we introduce $z=\sin ^{2}(\alpha \varphi)$ which brings Eq. (8) to the form

$$
\begin{aligned}
& \left\{\frac{\mathrm{d}^{2}}{\mathrm{~d} z^{2}}+\frac{\frac{1}{2}-z}{z(1-z)} \frac{\mathrm{d}}{\mathrm{d} z}+\frac{1}{z^{2}(1-z)^{2}}\left[-\frac{m^{2}}{4 \alpha^{2}} z^{2}+\left(\frac{m^{2}}{4 \alpha^{2}}+\frac{D(D-1)}{2}\left(M+E_{n, l, m}\right)-\frac{C(C-1)}{2}\left(M+E_{n, l, m}\right)\right) z\right.\right. \\
& \left.\left.-\frac{D(D-1)}{2}\left(M+E_{n, l, m}\right)\right]\right\} K_{m}(z)=0 .
\end{aligned}
$$

To solving Eq. (9), we use the NU method given compactly in Appendix A. Comparison of Eq. (9) with Eq. (A.1) reveals the correspondence

$$
\begin{aligned}
& \xi_{1}=\frac{m^{2}}{4 \alpha^{2}}, \quad \xi_{2}=\frac{m^{2}}{4 \alpha^{2}}+\frac{D(D-1)}{2}\left(M+E_{n, l, m}\right)-\frac{C(C-1)}{2}\left(M+E_{n, l, m}\right), \quad \xi_{3}=\frac{D(D-1)}{2}\left(M+E_{n, l, m}\right), \\
& \alpha_{1}=\frac{1}{2}, \quad \alpha_{2}=\alpha_{3}=1 .
\end{aligned}
$$

Thus, from Eq. (A.3), we simply find the other parameters as

$$
\begin{aligned}
& \alpha_{4}=\frac{1}{4}, \quad \alpha_{5}=-\frac{1}{2}, \quad \alpha_{6}=\frac{m^{2}}{4 \alpha^{2}}+\frac{1}{4}, \quad \alpha_{7}=-\frac{1}{4}-\frac{m^{2}}{4 \alpha^{2}}+\frac{M+E_{n, l, m}}{2}[-D(D-1)+C(C-1)], \\
& \alpha_{8}=\frac{1}{16}+\frac{D(D-1)\left(M+E_{n, l, m}\right)}{2}, \quad \alpha_{9}=\frac{1}{16}+\frac{C(C-1)\left(M+E_{n, l, m}\right)}{2}, \\
& \alpha_{11}=2+2\left(\sqrt{\frac{1}{16}+\frac{C(C-1)\left(M+E_{n, l, m}\right)}{2}}+\sqrt{\frac{1}{16}+\frac{D(D-1)\left(M+E_{n, l, m}\right)}{2}}\right), \\
& \alpha_{10}=1+2 \sqrt{\frac{1}{16}+\frac{D(D-1)\left(M+E_{n, l, m}\right)}{2}}, \quad \alpha_{12}=\frac{1}{4}+\sqrt{\frac{1}{16}+\frac{D(D-1)\left(M+E_{n, l, m}\right)}{2}}, \\
& \alpha_{13}=-\frac{1}{2}-\left(\sqrt{\frac{1}{16}+\frac{C(C-1)\left(M+E_{n, l, m}\right)}{2}}+\sqrt{\frac{1}{16}+\frac{D(D-1)\left(M+E_{n, l, m}\right)}{2}}\right) .
\end{aligned}
$$

As a result, Eqs. (A.2) and (A.3) give the energy relation as

$$
m^{2}=4 \alpha^{2}\left(n^{\prime}+\frac{1}{2}+\sqrt{\frac{1}{16}+\frac{C(C-1)\left(M+E_{n, l, m}\right)}{2}}+\sqrt{\frac{1}{16}+\frac{D(D-1)\left(M+E_{n, l, m}\right)}{2}}\right)^{2}, \quad n^{\prime}=0,1,2, \ldots
$$

The corresponding wave function is

$$
\begin{aligned}
& K_{m}(\varphi)=\left[\sin ^{2}(\alpha \varphi)\right]^{\frac{1}{4}+\sqrt{\frac{1}{16}+\frac{D(D-1)\left(M+E_{n, l, m}\right)}{2}}}\left(1-\sin ^{2}(\alpha \varphi)\right)^{\frac{1}{4}+\sqrt{\frac{1}{16}+\frac{C(C-1)\left(M+E_{n, l, m}\right)}{2}}} \\
& \quad \times P_{n^{\prime}}\left(2 \sqrt{\frac{1}{16}+\frac{D(D-1)\left(M+E_{n, l, m)}\right.}{2}}, 2 \sqrt{\frac{1}{16}+\frac{C(C-1)\left(M+E_{n, l, m}\right)}{2}}\right)\left(1-2 \sin ^{2}(\alpha \varphi)\right) .
\end{aligned}
$$




\subsection{Solution of the polar part}

Letting $x=\sin ^{2} \theta$, brings Eq. (7) into the form

$$
\begin{aligned}
& \left\{\frac{\mathrm{d}^{2}}{\mathrm{~d} x^{2}}+\frac{\frac{1}{2}-x}{x(1-x)} \frac{\mathrm{d}}{\mathrm{d} x}+\frac{1}{(x(1-x))^{2}}\left[-\frac{l^{2}}{4} x^{2}+\left(\frac{m^{2}+2 b\left(M+E_{n, l, m}\right)+l^{2}}{4}-\frac{1}{2} A(A-1)\left(M+E_{n, l, m}\right)-\frac{1}{16}\right) x\right.\right. \\
& \left.\left.\quad-\left(\frac{m^{2}+2 b\left(M+E_{n, l, m}\right)}{4}-\frac{1}{16}\right)\right]\right\} H_{l, m}(x)=0
\end{aligned}
$$

By the same token of the previous subsection, the eigenfunctions of the polar part are expressed in terms of the Jacobi polynomials as

$$
\begin{aligned}
& H_{l, m}(\theta)=\left(\sin ^{2} \theta\right)^{\frac{1}{4}+\frac{1}{2} \sqrt{m^{2}+2 b\left(M+E_{n, l, m}\right)}}\left(1-\sin ^{2} \theta\right)^{\frac{1}{4}+\sqrt{\frac{1}{16}+\frac{A(A-1)}{2}\left(M+E_{n, l, m}\right)}} \\
& \quad \times P_{n^{\prime \prime}}^{\left(\sqrt{m^{2}+2 b\left(M+E_{n, l, m}\right)}, 2 \sqrt{\frac{1}{16}+\frac{A(A-1)}{2}\left(M+E_{n, l, m}\right)}\right)}\left(1-2 \sin ^{2} \theta\right) .
\end{aligned}
$$

The energy relation in this case is

$$
l^{2}=4\left(n^{\prime \prime}+\frac{1}{2}+\sqrt{\frac{1}{16}+\frac{1}{2} A(A-1)\left(M+E_{n, l, m}\right)}+\frac{1}{2} \sqrt{m^{2}+2 b\left(M+E_{n, l, m}\right)}\right)^{2}, \quad n^{\prime \prime}=0,1, \ldots
$$

\subsection{Solution of the radial part}

Our starting square for the radial coordinate is

$$
-\frac{\mathrm{d}^{2} R_{n, l, m}(r)}{\mathrm{d} r^{2}}+\left[\left(l^{2}-\frac{1}{4}\right) \frac{1}{r^{2}}-2 \beta\left(M+E_{n, l, m}\right) \frac{1}{r}\right] R_{n, l, m}(r)=\left(E_{n, l, m}^{2}-M^{2}\right) R_{n, l, m}(r) .
$$

Again by using the NU method, we can find the energy eigenvalues equation and eigenfunctions as

$$
\begin{aligned}
& (2 n+1) \sqrt{M^{2}-E_{n, l, m}^{2}}-2 \beta(M+E)+2 l \sqrt{\left(M^{2}-E_{n, l, m}^{2}\right)}=0, \\
& R_{n, l, m}(r)=r^{\frac{1}{2}+l} \exp \left(-\sqrt{M^{2}-E_{n, l, m}^{2} r}\right) L_{n}^{2 l}\left(2 \sqrt{M^{2}-E_{n, l, m}^{2}} r\right) .
\end{aligned}
$$

As the final point, the total wave function is

$$
\begin{aligned}
& \psi(\boldsymbol{r})=N_{n, l, m} r^{-\frac{1}{2}+l} \exp \left(-\sqrt{M^{2}-E_{n, l, m}^{2}} r\right)\left(\sin ^{2} \theta\right)^{\frac{1}{4}+\frac{1}{2} \sqrt{m^{2}+2 b\left(M+E_{n, l, m}\right)}}\left(1-\sin ^{2} \theta\right)^{\frac{1}{4}+\sqrt{\frac{1}{16}+\frac{A(A-1)}{2}\left(M+E_{n, l, m}\right)}} \\
& \times\left[\sin ^{2}(\alpha \varphi)\right]^{\frac{1}{4}+\sqrt{\frac{1}{16}+\frac{D(D-1)\left(M+E_{n, l, m}\right)}{2}}}\left(1-\sin ^{2}(\alpha \varphi)\right)^{\frac{1}{4}+\sqrt{\frac{1}{16}+\frac{C(C-1)\left(M+E_{n, l, m}\right)}{2}}} L_{n}^{2 l}\left(2 \sqrt{\left.M^{2}-E_{n, l, m}^{2} r\right)}\right. \\
& \times P_{n^{\prime \prime}}^{\left(\sqrt{m^{2}+2 b\left(M+E_{n, l, m}\right)}, 2 \sqrt{\frac{1}{16}+\frac{A(A-1)}{2}\left(M+E_{n, l, m}\right)}\right)}\left(1-2 \sin ^{2} \theta\right) \\
& \quad \times P_{n^{\prime}}^{\left(2 \sqrt{\frac{1}{16}+\frac{D(D-1)\left(M+E_{n, l, m}\right)}{2}}, 2 \sqrt{\frac{1}{16}+\frac{C(C-1)\left(M+E_{n, l, m}\right)}{2}}\right)}\left(1-2 \sin ^{2}(\alpha \varphi)\right) .
\end{aligned}
$$

\section{Some special cases}

In this section, we are going to study two special cases of the Pöschl-Teller double-ring-shaped Coulomb potential and their corresponding eigenvalues and eigenfunctions by choosing approximate parameters in this potential.

\subsection{Hartman potential}

As we mentioned in Introduction, by choosing $A=C=D=1$, the Pöschl-Teller double-ring-shaped Coulomb potential turns into the Hartman potential

$$
V(r, \theta)=-\frac{\beta}{r}+\frac{b}{r^{2} \sin ^{2} \theta} .
$$

In this case we obtain the following equations for the energy eigenvalues and the corresponding eigenfunctions:

$$
\begin{aligned}
& (2 n+1) \sqrt{M^{2}-E_{n, l, m}^{2}}-2 \beta(M+E)+2 l \sqrt{M^{2}-E_{n, l, m}^{2}}=0, \\
& \psi(\boldsymbol{r})=N_{n, l, m} r^{-\frac{1}{2}+l} \exp \left(-\sqrt{M^{2}-E_{n, l, m}^{2} r}\right)\left(\sin ^{2} \theta\right)^{\frac{1}{4}+\frac{1}{2}} \sqrt{m^{2}+2 b\left(M+E_{n, l, m}\right)}\left(1-\sin ^{2} \theta\right)^{\frac{1}{2}} \\
& \left.\quad \times L_{n}^{2 l}\left(2 \sqrt{M^{2}-E_{n, l, m}^{2}} r\right) P_{n^{\prime \prime}}^{\left(\sqrt{m^{2}+2 b\left(M+E_{n, l, m}\right)}, \frac{1}{2}\right.}\right)\left(1-2 \sin ^{2} \theta\right) \mathrm{e}^{\mathrm{i} m \varphi},
\end{aligned}
$$

where we have 


$$
l^{2}=4\left(n^{\prime \prime}+\frac{3}{4}+\frac{1}{2} \sqrt{m^{2}+2 b\left(M+E_{n, l, m}\right)}\right)^{2} .
$$

\subsection{Double-ring-shaped Coulomb potential}

If we choose $C=D=1$, the Pöschl-Teller double-ring-shaped Coulomb potential changes into the double-ring-shaped Coulomb potential

$$
V(r, \theta)=-\frac{\beta}{r}+\frac{b}{r^{2} \sin ^{2} \theta}+\frac{A(A-1)}{r^{2} \cos ^{2} \theta} .
$$

The energy eigenvalues and the corresponding eigenfunctions of the latter have the form

$$
\begin{aligned}
& (2 n+1) \sqrt{M^{2}-E_{n, l, m}^{2}}-2 \beta(M+E)+2 l \sqrt{M^{2}-E_{n, l, m}^{2}}=0, \\
& \psi(\boldsymbol{r})=N_{n, l, m} r^{-\frac{1}{2}+l} \exp \left(-\sqrt{M^{2}-E_{n, l, m}^{2}} r\right)\left(\sin ^{2} \theta\right)^{\frac{1}{4}+\frac{1}{2} \sqrt{m^{2}+2 b\left(M+E_{n, l, m}\right)}}\left(1-\sin ^{2} \theta\right)^{\frac{1}{4}+\sqrt{\frac{1}{16}+\frac{A(A-1)}{2}\left(M+E_{n, l, m}\right)}} \\
& \quad \times L_{n}^{2 l}\left(2 \sqrt{M^{2}-E_{n, l, m}^{2}} r\right) P_{n^{\prime \prime}}^{\left(\sqrt{m^{2}+2 b\left(M+E_{n, l, m}\right)}, 2 \sqrt{\frac{1}{16}+\frac{A(A-1)}{2}\left(M+E_{n, l, m}\right)}\right)}\left(1-2 \sin ^{2} \theta\right) \mathrm{e}^{\mathrm{i} m \varphi},
\end{aligned}
$$

with

$$
l^{2}=4\left(n^{\prime \prime}+\frac{1}{2}+\sqrt{\frac{1}{16}+\frac{1}{2} A(A-1)\left(M+E_{n, l, m}\right)}+\frac{1}{2} \sqrt{m^{2}+2 b\left(M+E_{n, l, m}\right)}\right)^{2} .
$$

\section{Conclusion}

We obtained the exact solution of the radial and angular parts of the Klein-Gordon equation for Pöschl-Teller double-ring-shaped Coulomb potential using the NU method without having to do the cumbersome numerical steps. We have also studied some special cases of this potential. Our straightforward study can be simply understood even by the unfamiliar or undergraduate readers. Due to application of this potential in quantum chemistry and nuclear physics, our solutions can be used within these fields with the requisite modifications done.

\section{Appendix A}

The NU method solves many linear second-order differential equations by reducing them to a generalized equation of hypergeometric type. Here we use its parametric version which enables us to solve a second-order differential equation of the form [30, 32]:

$$
\left\{\frac{\mathrm{d}^{2}}{\mathrm{~d} s^{2}}+\frac{\alpha_{1}-\alpha_{2} s}{s\left(1-\alpha_{3} s\right)} \frac{\mathrm{d}}{\mathrm{d} s}+\frac{1}{\left[s\left(1-\alpha_{3} s\right)\right]^{2}}\left[-\xi_{1} s^{2}+\xi_{2} s-\xi_{3}\right]\right\} \psi=0 .
$$

According to the NU method, the eigenfunction is

$$
\psi(s)=s^{\alpha_{12}}\left(1-\alpha_{3} s\right)^{-\alpha_{12}-\frac{\alpha_{13}}{\alpha_{3}}} P_{n}^{\left(\alpha_{10}-1, \frac{\alpha_{11}}{\alpha_{3}}-\alpha_{10}-1\right)}\left(1-2 \alpha_{3} s\right),
$$

and the energy of the system satisfies

$$
\alpha_{2} n-(2 n+1) \alpha_{5}+(2 n+1)\left(\sqrt{\alpha_{9}}+\alpha_{3} \sqrt{\alpha_{8}}\right)+n(n-1) \alpha_{3}+\alpha_{7}+2 \alpha_{3} \alpha_{8}+2 \sqrt{\alpha_{8} \alpha_{9}}=0
$$
where

$$
\begin{aligned}
& \alpha_{4}=\frac{1}{2}\left(1-\alpha_{1}\right), \quad \alpha_{5}=\frac{1}{2}\left(\alpha_{2}-2 \alpha_{3}\right), \quad \alpha_{6}=\alpha_{5}^{2}+\xi_{1}, \quad \alpha_{7}=2 \alpha_{4} \alpha_{5}-\xi_{2}, \quad \alpha_{8}=\alpha_{4}^{2}+\xi_{3}, \quad \alpha_{9}=\alpha_{3} \alpha_{7}+\alpha_{3}^{2} \alpha_{8}+\alpha_{6}, \\
& \alpha_{10}=\alpha_{1}+2 \alpha_{4}+2 \sqrt{\alpha_{8}}, \quad \alpha_{11}=\alpha_{2}-2 \alpha_{5}+2\left(\sqrt{\alpha_{9}}+\alpha_{3} \sqrt{\alpha_{8}}\right), \\
& \alpha_{12}=\alpha_{4}+\sqrt{\alpha_{8}}, \quad \alpha_{13}=\alpha_{5}-\left(\sqrt{\alpha_{9}}+\alpha_{3} \sqrt{\alpha_{8}}\right), \\
& P_{n}^{(\alpha, \beta)}(x)=\frac{\Gamma(\alpha+n+1)}{n ! \Gamma(\alpha+\beta+n+1)} \sum_{m=0}^{n}\left(\begin{array}{c}
n \\
m
\end{array}\right) \frac{\Gamma(\alpha+\beta+n+m+1)}{\Gamma(\alpha+m+1)}\left(\frac{x-1}{2}\right)^{m},
\end{aligned}
$$

and $P_{n}^{(\alpha, \beta)}$ is the Jacobi polynomial.

\section{Acknowledgments}

We wish to express our sincere gratitude to the referee for his invaluable comments.

\section{References}

[1] G. Pöschl, E. Teller, Z. Phys. 83, 143 (1933).

[2] M. Simsek, Z. Yalcin, J. Math. Chem. 16, 211 (1994). 
[3] M. Aktas, R. Sever, J. Mol. Struct. Theochem. 710, 223 (2004).

[4] J. Zuniga, M. Alacid, A. Requena, A. Bastida, Int. J. Quant. Chem. 57, 43 (1995).

[5] J.M. Arias, J. Gomez Camacho, R. Lemus, J. Phys. A: Math. Gen. 37, 877 (2004).

[6] J.I. Daz, J. Negro, L.M. Nieto, O. Rosas, J. Phys. A 32, 8447 (1999).

[7] M. Boca, M. Stroe, Rom. Rep. Phys. 55, 184 (1998).

[8] M. Znojil, Phys. Lett. A 266, 4 (2000).

[9] R. da Rocha, E. Capelas de Oliveira, Rev. Mex. Fis. 51, 1 (2005).

[10] E. Capelas de Oliveira, R. da Rocha, Electron. J. Theor. Phys. 18, 21 (2008).

[11] R. Koc, M. Koca, arXiv:math-ph 0505004.

[12] B. Bagchi, A. Ganguly, J. Phys. A, Math. Gen. 36, 161 (2003)

[13] L.D. Landau, E.M. Lifshitz, Quantum Mechanics, 3rd ed., Pergamon, London 1965.

[14] C.S. Jia, P. Guo, Y.F. Diao, L.Z. Yi, X.J. Xie, Eur. Phys. J. A 34, 41 (2007).

[15] Y. Xu, H. Su, C.S. Jia, J. Phys. A, Math. Theor. 41, 255302 (2008).

[16] C.S. Jia, T. Chen, L.G. Cui, Phys. Lett. A 373, 1621 (2009).

[17] Y. Xu, S. He, C.S. Jia, Phys. Scr. 81, 045001 (2010).

[18] Y. Hasan, M. Tomak, Phys. Rev. B 72, 115340 (2005).

[19] A. Rodríguez, J. Cerveró, Phys. Rev. B 74, 104201 (2006).

[20] U. Roy, S. Ghosh, P.K. Panigrahi, D. Vitali, Phys. Rev. A 80, 052115 (2009).

[21] H. Yildirim, M. Tomak, J. Appl. Phys. 99, 093103 (2006).

[22] W. Xie, Commun. Theor. Phys. 46, 1101 (2006).

[23] H. Hassanabadi, B.H. Yazarloo, S. Zarrinkamar, A.A. Rajabi, Phys. Rev. C 84, 064003 (2011).
[24] S.H. Dong, Z.Q. Ma, J. Phys. A 31, 9855 (1998).

[25] B.H. Yazarloo, H. Hassanabadi, S. Zarrinkamar, Eur. Phys. J. Plus 127, 51 (2012).

[26] G.F. Wei, S.H. Dong, Europ. Phys. Lett. 87, 40004 (2009).

[27] S. Hassanabadi, A.A. Rajabi, B.H. Yazarloo, S. Zarrinkamar, H. Hassanabadi, Advances in High Energy Physics 2012, 804652 (2012).

[28] S.H. Dong, Int J. Theor. Phys. 39, 1119 (2000).

[29] L.L. Lu, B.H. Yazarloo, S. Zarrinkamar, G. Liu, H. Hassanabadi, Few-Body Syst. 53, 573 (2012).

[30] G.F. Wei, S.H. Dong, Europ. Phys. Lett. 87, 40004 (2009).

[31] A.D. Antia, A.N. Ikot, I.O. Akpan, O.A. Awoga, Indian J. Phys. 87, 155 (2013).

[32] H. Hassanabadi, B.H. Yazarloo, S. Zarrinkamar, H. Rahimov, Commun. Theor. Phys. 57, 339 (2012).

[33] H. Hassanabadi, B.H. Yazarloo, L.L. Lu, Chin. Phys. Lett. 29, 020303 (2012).

[34] A.F. Nikiforov, V.B. Uvarov, Special Functions of Mathematical Physics, Birkhauser, Bassel 1988.

[35] W.C. Qiang, S.H. Dong, Phys. Lett. A. 368, 13 (2007).

[36] C. Tezcan, R. Sever, Int. J. Theor. Phys. 48, 337 (2009).

[37] S.H. Dong, M. Lozada-Cassou, Phys. Scr. 74, 285 (2006).

[38] G. Wei, S.H. Dong, Phys. Lett. A 373, 2428 (2009).

[39] S.H. Dong, Wave Equations in Higher Dimensions, Springer, Berlin 2011.

[40] F.L. Lu, C.Y. Chen, Chin. Phys. B 19, 100309 (2010).

[41] B.W. Huang, High Energy Phys. Nucl. Phys. 27, 770 (2003)

[42] F. Yasuk, A. Durmus, Phys. Scr. 77, 015005 (2008). 